\title{
Editorial \\ Lipooxygenase inhibition in osteoarthritis: a potential symptomatic and disease modifying effect?
}

Maxime Dougados

Paris-Descartes University, Medicine Faculty; UPRES-EA 4058; AP-HP, Cochin Hospital, Rheumatology B Department, Paris, France

Corresponding author: Maxime Dougados, maxime.dougados@cch.aphp.fr

Published: 19 September 2008

Arthritis Research \& Therapy 2008, 10:116 (doi:10.1186/ar2490)

This article is online at http://arthritis-research.com/content/10/5/116

(c) 2008 BioMed Central Ltd

See related research article by Sengupta et al., http://arthritis-research.com/content/10/4/R85

\begin{abstract}
5-Loxin ${ }^{\circledR}$ is a compound extracted from an ancient herb that might be considered as a potent lipooxygenase inhibitor. The results observed in a preliminary trial suggest a highly intriguing clinically relevant symptomatic effect contrasting with a poor bioavailability of this compound. Moreover, in this trial, a statistically significant decrease in matrix metalloproteinase enzyme serum level suggests that, in addition to the observed symptomatic effect, such a compound might have a disease modifying effect in osteoarthritis. Further studies are required both to confirm the symptomatic efficacy and acceptable safety profile and to evaluate the potential chondromodulating effect of this compound.
\end{abstract}

In the previous issue of Arthritis Research \& Therapy, Sengupta and colleagues [1] report the results of a shortterm placebo randomized controlled trial evaluating both the symptomatic and biological effects of the compound 5Loxin $^{\circledR}$, which is a gum resin extracted from an ancient herb. Despite the small sample size (25 patients per arm) and the short duration of this trial (3 months), the reported results are interesting and intriguing for several reasons.

In the field of rheumatology, most new therapeutic compounds are based on our knowledge of the pathophysiological pathways of the disease (for example, targeted therapies against cytokines such as tumor necrosis factor (TNF), interleukin-6, and so on) or against some specific enzyme (for example, cyclooxygenases). The compound evaluated in the trial reported by Sengupta and colleagues [1] is in fact derived from an ancient herb, Boswella. Because of the empirically observed anti-arthritic and analgesic effects of this compound, researchers have tried to understand its mechanism of action. In vitro data suggest that 5 -Loxin ${ }^{\circledR}$ might be considered as a potent lipooxygenase inhibitor but also, at a lower magnitude, an inhibitor of the TNF $\alpha$-induced gene expression of matrix metalloproteinases. Such in vitro findings have to be kept in mind when interpreting the results of the trial. In particular, should we consider that the observed symptomatic treatment effect is related to the lipooxygenase inhibition, to the anti-TNF $\alpha$ inhibition, to both, or to an as yet unknown mechanism?

This trial has been conducted in India. The researchers have to be congratulated because of the high quality of both the design, the conduct, the analysis and the reporting of the trial.

The observed symptomatic placebo effect is of a similar magnitude as those observed in previous placebo randomized controlled trials in this condition [2,3]. The observed symptomatic effect (for example, the differences in the changes in the active minus the placebo groups) seems to be not only statistically relevant but also highly clinically relevant. In osteoarthritis, a symptomatic treatment effect of a least 10 on a scale of 0 to 100 is usually considered as clinically relevant [4]. In this reported trial, a treatment effect of 26 (for the VAS [Visual Analogue Scale] score), 18 (for the WOMAC [Western Ontario and MacMaster universities osteoarthritis questionnaire] pain subscale) and 15 (for the WOMAC function sub-scale) were observed in the group of patients taking the compound at a $250 \mathrm{mg}$ daily dose when compared to placebo. Moreover, a trend in favor of a dose ranging effect (when comparing placebo, 5-Loxin ${ }^{\circledR} 100 \mathrm{mg}$ and 5-Loxin ${ }^{\circledR}$ $250 \mathrm{mg}$ ) supports the relevance of these results. These results are intriguing since the oral bioavailability of the active ingredient of 5-Loxin ${ }^{\circledR}$ is very low [5]. Such findings concerning the symptomatic effect of this compound have now to be confirmed in larger trials conducted in different parts of the world.

More intriguing are the results observed for some biological markers, especially matrix metalloproteinase-3 serum levels.

$\mathrm{TNF}=$ tumor necrosis factor 
Such findings raise the question of whether we should consider this compound as an alternative to the currently available symptomatic drugs (for example, analgesics, cyclooxygenase inhibitors, and so on) because of potentially better efficacy and safety profiles, or whether we have to consider that, in addition to its symptomatic effect, such a compound might have an osteoarthritic disease modifying effect. The demonstration of a disease modifying effect requires longterm studies with hard end-points, such as the capacity to reduce the requirement for total articular replacement, to reduce cartilage loss, and so on. In this study, the researchers have evaluated an interesting surrogate biomarker in this area, for example, the level of matrix metalloproteinase-3, which is deeply involved in the degradation of cartilage [6].

In conclusion, because of obvious unmet needs in the treatment of osteoarthritis and because we have yet to develop an efficient and safe targeted therapy, the reported results observed with this new compound are very promising, although they need to be confirmed in other studies evaluating not only its symptomatic effect but also its potential chondromodulating effect.

\section{Competing interests}

The author declares that they have no competing interests.

\section{References}

1. Sengupta K, Alluri KV, Sathis AR, Mishra S, Golakoti T, Sarma KVS, Dey D, SP Raychaudhuri: A double blind, randomized, placebo controlled study of the efficacy and safety of 5Loxin ${ }^{\circledR}$ for treatment of osteoarthritis of the knee. Arthritis Res Ther 2008, 10:R85.

2. Dougados $M$, Leclaire $P$, van der Heijde D, loch DA, Bellamy N, Altman RD: Response criteria for clinical trials on osteoarthritis of the knee and hip: a report of the OsteoArthritis Research Society International Standing Committee for Clinical Trials response criteria initiative. Osteoarthritis Cartilage 2000, 8:395-403.

3. Pham T, van der Heijde D, Altman RD, Anderson JJ, Bellamy N, Hochberg M, Strand V, Woodworth T, Dougados M: OMERACTOARSI initiative: Osteoarthritis Research Society International set of responder criteria for osteoarthritis clinical trials revisited. Osteoarthritis Cartilage 2004, 12:389-399.

4. Dougados M, Moore A, Yu S, Gitton X: Evaluation of the patient acceptable symptom state in a pooled analysis of two multicentre, randomized, double-blind, placebo-controlled studies evaluating lumiracoxib and celecoxib in patients with osteoarthritis. Arthritis Res Ther 2007, 9:R11.

5. Buchele B, Zugmaier W, Genze F, Simmet TJ: High-performance liquid chromatographic determination of acetyl-11alpha-boswellic acid, a novel pentacyclic triterpenoid, in plasma using a fluorinated stationary phase and photodiode array detection: application in pharmacokinetic studies. Chromatogr B Analyt Technol Biomed Life Sci 2005, 289:144-148

6. Pelletier JP, Martel-Pelletier J : DMOAD developments: present and future. Bull NYU Hosp Jt Dis 2007, 65:242-248. 